\title{
S.R. POTTASCH
}

\section{ABSTRACT :}

Determination of the distances to individual planetary nebulae are discussed. Especially those methods which are independent of assumed nebular properties (mass, absolute flux, etc.) are assembled and discussed. In this way, reasonable approximations to the distance can be obtained for about 50 planetary nebulae. The accuracy of the distances is tested by comparing nebular properties derived from these distances with the properties of nebulae at the galactic center or in the Magellanic clouds. A comparison is also made with the statistical distance determinations; the conclusion is that the assumption of constant mass often leads to an overestimate of the distance, while the assumption of constant $\mathrm{H} \beta$ flux leads to distances having individual uncertainties of up to a factor of 3 .

The central star temperature determination is summarized. Individual central stars are placed on the HR diagram and compared with theoretical predictions. Deductions concerning the evolution which can be made from the observations are discussed.

\section{A. INTRODUCTION}

In placing individual stars on the HR diagram the greatest uncertainties are the distance and the temperature of the star. Both of these problems will be discussed in turn, with emphasis on the distance because of the difficulties involved in its determination.

The usual methods of distance determination in astronomy, triginometric parallax and/or spectroscopic parallax, are seldom applicable to planetary nebula. Therefore methods have been devised for distance determination which are based on assumed nebular properties. The method in most common use is the so-called 'Shklovskii method', which assumes all nebulae have the same ionized mass. A second method assumes all nebulae have the same absolute $\mathrm{H} \beta$ flux. There is growing evidence that these assumptions are both incorrect. In fact they may have systematic errors affecting large and small nebulae in different ways. 
At present there are several methods available to determine individual distances, independent of any assumptions concerning the physical properties of the nebula. These distances give independent information on the properties of the nebulae, especially mass and density. These resulting values are then compared with those determined for nebulae found in the galactic center, whose distance is well established. This data allows an interesting comparison with the results of the constant ionized mass and constant absolute $\mathrm{H} \beta$ flux methods.

\section{B. DISTANCES TO INDIVIDUAL NEBULAE}

Seven methods are considered as independent ways of determining the distance. Some of the methods are more reliable than others, most have a limited application. We shall discuss each of the methods, and give some results for the more reliable methods.

(1) Spectroscopic parallax. Some planetary nebulae are excited by stars which have binary companions. If the spectral type and luminosity class of the companion can be measured, a spectroscopic distance for the system can be determined. This method may have a wide application, since it is estimated that at least $10 \%$ of the exciting stars are binaries. The method may be applied both to visual binaries (where both stars are seen separately) and to spectroscopic binaries where the 'normal' star dominates the spectrum. At present, however, only a very limited number of such cases have been well studied. The resultant distances are shown in Table 1; the nebulae are all nearby. This is consistent with the fact that the nebulae all have large angular diameters.

TABLE 1 - SPECTROSCOPIC DISTANCES

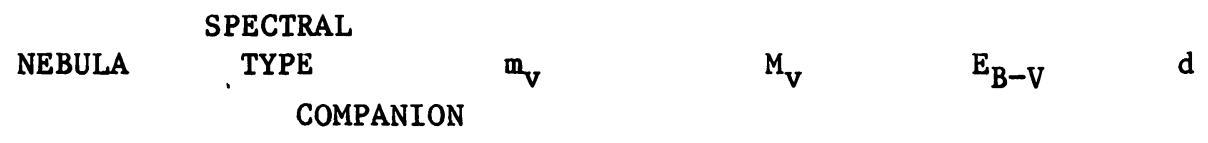

\begin{tabular}{|c|c|c|c|c|c|c|}
\hline $\begin{array}{c}\text { NGC } 246 \\
1514 \\
2346 \\
3132 \\
\text { A35 }\end{array}$ & $\begin{array}{l}\text { KO } \\
\text { A0 } \\
\text { A2 } \\
\text { A0 } \\
\text { G8 }\end{array}$ & $\begin{array}{l}V \\
I I I \\
V \\
V \\
I I I-I V\end{array}$ & $\begin{array}{r}14.3 \\
9.42 \\
11.12 \\
10.06 \\
9.63\end{array}$ & $\begin{array}{r}5.9 \\
-0.2 \\
1.4 \\
0.7 \\
1.9\end{array}$ & $\begin{array}{l}0.01 \\
0.45 \\
0.22 \\
0.07 \\
0\end{array}$ & $\begin{array}{l}470 \mathrm{pc} \\
400 \\
640 \\
670 \\
360\end{array}$ \\
\hline
\end{tabular}

The spectral types and magnitudes are taken from Lutz (1977), Mendez (1978), Jacoby (1981), and Lutz (1978).

(2) Expansion distances. Radial velocity measurements of the nebulae show a splitting of emission lines. This is interpreted as expansion of the nebulae, with velocities typically of $20 \mathrm{~km} \mathrm{~s}^{-1}$. One may compare this expansion velocity with angular expansions derived from comparing the location of knots, filaments edges, and other features seen on both old and new photographs. If spherical symmetry 
obtains, then the distance calculation is straight forward, and is given as follows

$$
\mathrm{d}=\frac{100 \mathrm{v}}{4.74 \theta} \mathrm{pc}
$$

where the distance $d$ is in pc, $\theta$ is the angular expansion in arc sec per 100 years, and $v$ is the measured radial velocity $\left(\mathrm{km} \mathrm{s}^{-1}\right)$.

Angular expansion rates have been determined by a number of investigators (see the references given under Table 2). The rates are difficult to measure, partly because of non-uniform shrinkage of the plate emulsion and partly because of the difficulty of finding sharply defined features to measure. The interpretation also has uncertainties. Firstly, the angular expansion may occur in a part of the nebula that is moving at a velocity different than the observed radial velocity. Secondly, expansions measured at the edge of optically thick nebulae may be influenced by motion of the ionization front, in addition to bulk motion of the gas. This second problem may be avoided if a well defined feature is measured.

Measurements of angular expansion rates and resultant distances are given in Table 2. Where two values are given for an individual nebula they are from different observers.

TABLE 2 - ANGULAR EXPANSION RATE AND RESULTANT DISTANCES

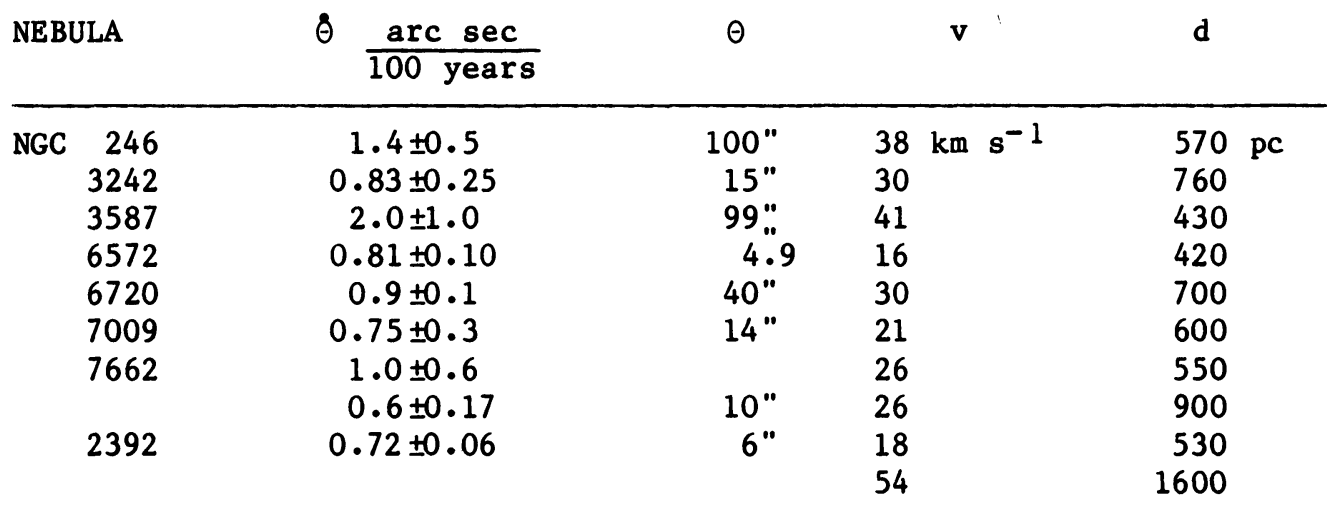

Angular expansion rates from Latypov (1955), Chudovicheva (1964), Liller et al., (1966), Liller and Liller (1968).

(3)

Distance determined on the basis of membership in a stellar group. Other than the planetary nebulae near the galactic center, or in an extragalactic system, there is only one well established case of a nebula which is a member of a stellar group. That is the planetary nebula discovered by Pease (Ps-1) in the globular cluster M15, in 1928 . The distance of M15 is given as 10.1 kpc (Harris, 1976).

(4) Interstellar extinction distances. This method is simple: by measuring the spectral type and $B$ and $V$ magnitudes of field stars close to the nebula in the plane of the sky, one can obtain both their 
distance and their color excess $\left(E_{B-V}\right)$. These two quantities should define a monotonic curve on which the planetary nebula may also be placed. If $E_{B-V}$ is known for the nebula, its distance follows directly.

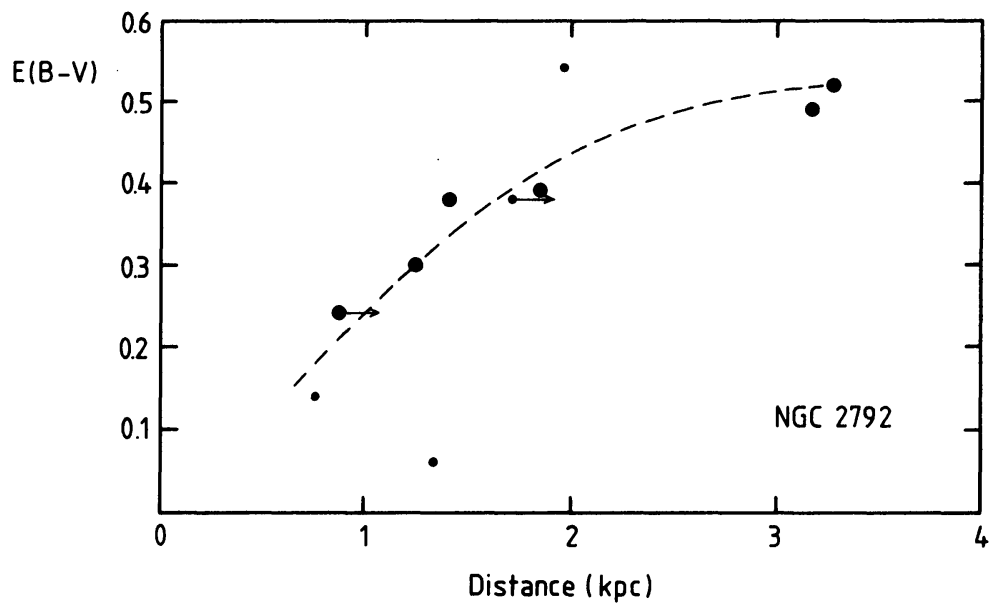

Fig. 1

$E_{B-V}$ plotted against distance d for field stars in the direction of the nebula NGC 2792. The color excess of the nebula is about 0.50 , leading to a distance of $2.7 \pm 0.5 \mathrm{Kpc}$.

An example of one such curve is shown in Fig. 1. The method has been used, as well for objects other than nebulae, e.g. novae. It is generally considered reliable if carefully applied. A summary of the method and details of the results to 1976 is given by Acker (1978).

This method can be applied to all nebulae with measurable extinction that are reasonably close to the galactic plane. There is no further limitation in principle, although in practice several problems arise because:

A. An insufficient number of measurements of individual stars close to a given nebula have been made. This requires using measurements as far as $5^{\circ}$ from the nebula, which often introduces errors because of the patchiness of the interstellar extinction.

B. Determining a correct distance to the field stars in the line-ofsight requires that their absolute magnitude be known. This can be determined from the spectrum, and sometimes, if one is careful, from the photometric colors as well. The limiting magnitudes for accurate spectroscopy and photometry consequently limit the method to moderately nearby nebulae. Only now are sufficiently accurate measurements becoming available (Acker, 1978; Gathier and Pottasch, 1983).

In a similar, but less accurate way, distances for a much larger number of nebulae can be determined. From the photometric and spectroscopic data in astronomical catalogues one can make distance vs. $\mathrm{E}_{\mathrm{B}-\mathrm{V}}$ plots in many areas of the sky. Since a sufficient number of stars is usually not available in a small region, average values of the extinction vs. distance over larger areas of the sky must be used.

Lucke (1978) has made such plots (see also Acker, 1976). It is not ideal for our purposes because the actual extinction shows variations on a scale considerably smaller than is shown in the diagram. These variations have been averaged out in the diagram. On the other hand, general trends in the extinction can be clearly seen and give a useful 
first approximation to the distance. At distances greater than 300 to 500 pc above the plane, there is probably very little extinction and the method is of little use for nebulae this far the plane.

In Table 3, distances for more than 60 nebulae close to the galactic plane are given. One can judge the accuracy by comparing them with the more accurate (but preliminary) results of Gathier and Pottasch and the results of other methods. It appears that the accuracy is usually better than a factor of 2 .

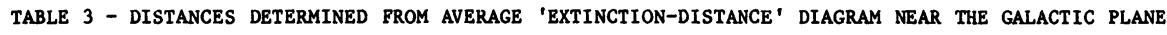

\begin{tabular}{|c|c|c|c|c|c|c|c|c|c|c|c|}
\hline NEBULA & P.K. No. & $\mathrm{E}_{\mathrm{B}-\mathrm{V}}$ & d & NEBULA & P.K. No. & $\mathrm{E}_{\mathrm{B}-\mathrm{V}}$ & d & NEBULA & P.K. No. & $\mathrm{E}_{\mathrm{B}-\mathrm{V}}$ & d \\
\hline $\begin{array}{cc}\text { N } & 40 \\
\text { IC } & 1747 \\
\text { N } & 1501 \\
\text { N } & 2022 \\
\text { IC } & 2149 \\
\text { IC } & 2165 \\
\text { J } & 900 \\
\text { N } & 2346 \\
\text { N } & 2438 \\
\text { N } & 2440 \\
\text { N } & 2452 \\
\text { N } & 2792 \\
\text { N } & 2867 \\
\text { N } & 2818 \\
\text { N } & 3211 \\
\text { N } & 3918 \\
\text { N } & 5882\end{array}$ & $\begin{array}{l}120+9^{\circ} 1 \\
130+1^{\circ} 1 \\
144+6^{\circ} 1 \\
196-10^{\circ} 1 \\
166+10^{\circ} 1 \\
221-12^{\circ} 1 \\
194-2^{\circ} 1 \\
215+3^{\circ} 1 \\
231+4^{\circ} 2 \\
234+2^{\circ} 1 \\
243-1^{\circ} 1 \\
265+4^{\circ} 1 \\
278-5^{\circ} 1 \\
261+8^{\circ} 1 \\
286-4^{\circ} 1 \\
294+4^{\circ} 1 \\
327+10^{\circ} 1\end{array}$ & $\begin{array}{l}0.50 \\
0.67 \\
0.74 \\
0.26 \\
0.31 \\
0.38 \\
0.56 \\
0.20 \\
0.29 \\
0.31 \\
0.45 \\
0.57 \\
0.28 \\
0.20 \\
0.22 \\
0.28 \\
0.30\end{array}$ & $\begin{array}{l}0.8 \mathrm{kpc} \\
3.0 \\
1.4 \\
1.3 \\
1.2 \\
1.9 \\
2.0: \\
0.9 \\
2.0 \\
1.6 \\
3.0 \\
2.5 \\
2.0 \\
1.8: \\
2.5 \\
1.3 \\
1.3\end{array}$ & $\begin{array}{cc}N & 5315 \\
N & 6072 \\
N & 5189 \\
N & 6153 \\
N & 6326 \\
N & 6439 \\
N & 6369 \\
N & 6445 \\
N & 6565 \\
N & 6563 \\
N & 6567 \\
N & 6572 \\
N & 6629 \\
N & 6720 \\
N & 6751 \\
N & 6741 \\
N & 6772\end{array}$ & $\begin{array}{r}309-4^{\circ} 2 \\
342+10^{\circ} 1 \\
307-3^{\circ} 1 \\
341+5^{\circ} 1 \\
338-8^{\circ} 1 \\
11+5^{\circ} 1 \\
2+5^{\circ} 1 \\
8+3^{\circ} 1 \\
3-4^{\circ} 5 \\
358-7^{\circ} 1 \\
11-0^{\circ} 2 \\
34+11^{\circ} 1 \\
9-5^{\circ} 1 \\
63+13^{\circ} 1 \\
29-5^{\circ} 1 \\
33-2^{\circ} 1 \\
33-6^{\circ} 1\end{array}$ & $\begin{array}{l}0.42 \\
0.69 \\
0.40 \\
0.71 \\
0.25 \\
0.53 \\
1.43 \\
0.83 \\
0.30 \\
0.23 \\
0.48 \\
0.29 \\
0.66 \\
0.07 \\
0.50 \\
0.83 \\
0.73\end{array}$ & $\begin{array}{l}1.3 \mathrm{kpc} \\
1.8 \\
0.8 \\
1.8 \\
0.8 \\
1.3 \\
1.5 \\
2.5 \\
1.3 \\
0.8 \\
1.3 \\
0.7 \\
1.6 \\
0.35 \\
1.5 \\
1.4 \\
1.3\end{array}$ & \begin{tabular}{|cc|c}
$N$ & 6778 \\
$N$ & 6790 \\
$N$ & 6803 \\
$N$ & 6804 \\
BD +30 \\
He2-131 \\
N & 6853 \\
N & 6884 \\
N & 6886 \\
N & 6894 \\
N & 7008 \\
N & 7026 \\
Hu & $1-2$ \\
IC & 5217 \\
N & 6578 \\
$N$ & 6781 \\
IC & 5117
\end{tabular} & $\begin{array}{r}34-6^{\circ} 1 \\
37-6^{\circ} 1 \\
46-4^{\circ} 1 \\
45-4^{\circ} 1 \\
64+5^{\circ} 1 \\
315+13^{\circ} 1 \\
60-3^{\circ} 1 \\
82+7^{\circ} 1 \\
60-7^{\circ} 2 \\
69-2^{\circ} 1 \\
93-5^{\circ} 2 \\
89+0^{\circ} 1 \\
86-8^{\circ} 1 \\
100-5^{\circ} 1 \\
10-1^{\circ} 1 \\
41-2^{\circ} 1 \\
89-5^{\circ} 1\end{array}$ & $\begin{array}{l}0.23 \\
0.60 \\
0.48 \\
0.62 \\
0.24 \\
0.18 \\
0.05 \\
0.68 \\
0.58 \\
0.50 \\
0.50 \\
0.65 \\
0.45 \\
0.45 \\
1.02 \\
0.85 \\
0.87\end{array}$ & $\begin{array}{l}1.0 \mathrm{kpc} \\
1.2 \\
1.7 \\
2.0 \\
0.6 \\
1.0 \\
0.25 \\
2.0 \\
2.0 \\
1.5 \\
1.1 \\
2.3 \\
1.5 \\
1.5 \\
2.2 \\
1.5 \\
2.5\end{array}$ \\
\hline
\end{tabular}

(5)

Comparison of 'forbidden line' $n_{e}$ with 'recombination line flux measurement. The $\mathrm{H} \beta$ flux depends on density, nebular angular size, filling factor and distance. If the density is known from forbidden line ratio, the angular size is measured and the filling factor can be estimated from the observed geometry, the distance can be found. In practice the method gives unreliable results. There may be several reasons for this. First of all, the density is difficult to determine with sufficient accuracy from the forbidden lines, possibly because of variations within the nebula. Since the distance depends on the square of the density, an accurate value is required. Secondly, the geometry is often difficult to define with sufficient accuracy. The distance depends on the cube of the radius, which may be difficult to define precisely, either because of irregular structure or faint outer emission. Since the method is unreliable it is not of general interest. It may still be used as a check on the distances determined from other methods, as well as a means of gaining insight about the filling factor (see section $C$ below).

(6) Stellar atmosphere analysis. Recently attempts have been made to explain the absorption line profiles observed in the spectra of some central stars. The models used predict the effective temperature and surface gravity of the star. The gravity, when coupled with an assumed stellar mass gives a value of the stellar radius. When these two values are combined with the measured visual magnitude of the star, the distance can be found.

The method can only be applied to stars which have an absorption line spectrum. Model atmospheres have been constructed for 5 cen- 
tral stars by Kudritzki et al. (1981). The method is in an early stage, and present accuracy is probably not better than a factor of 3 but it appears to be a promising approach for a limited number of stars.

(7) $21 \mathrm{~cm}$ hydrogen absorption line measurements. For nebulae located close to the galactic plane, with radio continuum fluxes greater than $100 \mathrm{mJy}$ (at $21 \mathrm{~cm}$ ), it is now possible to observe the $21 \mathrm{~cm}$ interstellar hydrogen absorption line. The strength of the absorption line is a measure of the distance. In this respect the method is similar to the extinction method. Another similarity is the necessity for calibrating the absorption - distance relation in different directions. Absorption line profiles give velocity information as well as line strength. Individual absorption profiles often correspond to a known spiral arm farther than the local arm. If the distance of the arm is known from other data, a lower limit for the distance of the planetary nebula can be obtained. If in addition the $21 \mathrm{~cm}$ absorption line is also measured in a nearby extragalactic source, it may be possible to pinpoint whether the nebula is located in or beyond the spiral arm.

As an example, Fig. 2 shows the 21 absorption profile of NGC 6537. The local arm and the Sagittarius arm, about $1.5 \mathrm{Kpc}$ distant, are clearly evident. The Scutum arm $3.5 \mathrm{Kpc}$ distant is completely absent. Hence the nebula must be at a distance of 2 to $3 \mathrm{Kpc}$. This method of distance determination has only recently been applied to planetary nebulae (Pottasch et al., 1981, 1983), although it has been used earlier for other difficult objects such as supernova remnants and pulsars.

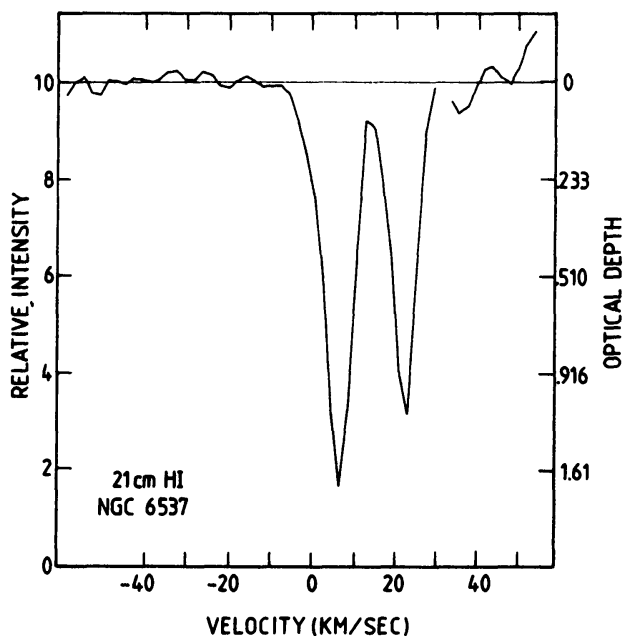

$\underline{F i g \cdot 2}$

The $21 \mathrm{~cm}$ absorption line profile in the direction of NGC 6537.

Absorption due to the local arm and the Sagittarius arm are clearly seen, but no evidence for a further arm is present.

\section{MASS AND DENSITY OF NEARBY NEBULAE.}

When the distance is known it is possible to compare the various nebulae with each other, and especially to compare the nearby nebulae with those near the galactic center. To do this, the mass $M$ and density $n_{\text {rms }}$ are computed and plotted against each other as explained below. 
This computation is done with a simple model: a sphere of uniform density $n_{e}$, with the material in clumps which fill a fraction $\varepsilon$ of the total volume. The temperature $T_{e}$ is also constant. The density and mass are then given by

$$
\begin{aligned}
& \mathrm{n}_{\mathrm{e}} \varepsilon^{1 / 2}=2.74 \times 10^{4}\left(\frac{\mathrm{F}_{\mathrm{H} \beta^{t^{0}} \cdot 88}}{\theta^{3} \mathrm{~d}}\right)^{1 / 2} \mathrm{~cm}^{-3} \\
& M=11.06 F_{H \beta} d^{2} t^{0.88} n_{e} e^{-1} M_{\odot}
\end{aligned}
$$

where $d$ is the distance in $\mathrm{Kpc}, \mathrm{F}_{\mathrm{H} \beta}$ the $\mathrm{H} \beta$ flux corrected for extinction in units of $10^{-11}$ erg $\mathrm{cm}^{-2} \mathrm{~s}^{-1}$. The angular radius $\theta$ is in arc sec and $t=10^{4} T_{e} \cdot M$ is quite strongly distance dependent $(5 / 2$ power) while $\mathrm{n}_{e}$ is much less dependent ( $1 / 2$ power).

Once the distance has been determined, all the quantities in the above equations are specified, except the filling factor $\varepsilon$. To find an average value for $\varepsilon, n_{e}$ has been determined from equation (1), assuming $\varepsilon=1$. This density, called $n_{\text {rms }}$, may then be plotted against the density $n_{e}$ determined for each nebula from the ratio of forbidden lines. Such a plot is shown as Fig. 3, where it can be seen that there is good agreement (close to the line $\varepsilon=1$ ) between the two values. Thus $\varepsilon=1$ is an acceptable value and there are no systematic departures from it. At first glance this is surprising, since examination of photographs of most of the (larger) nebulae show the presence of structure on different size scales. The answer may be: (1) the dissymmetry of the nebulae as a whole is at least partly taken into account in the determination of the angular radius, and (2) photographs

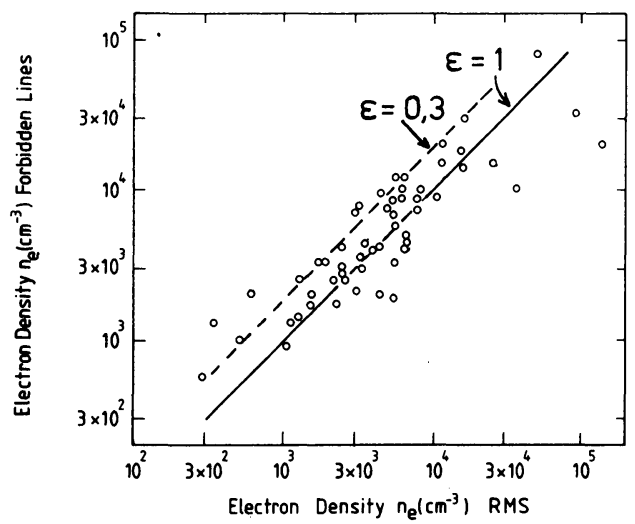

$\underline{\text { Fig. } 3}$

The rms density obtained from eq.(1) is plotted against the electron density obtained from the ratio of forbidden lines for all nebulae for which distances could be found. A one to one correspondence is found, indicating that the filling does not systematically depart from unity.

exaggerate the importance of the smaller scale structure. On the other hand, nebulae with very low $n_{\text {rms }}$ have not been included in Fig. 3, since no reliable forbidden line densities are available.

The ionized mass $M$ and electron density $n_{e}$ may now be determined, and are plotted against each other in Fig. 4. The names of the individual nebulae are given in the figure. From inspection of the figure, several conclusions can be drawn. 
a) There is a wide range of lonized mass, from less than $10^{-4} M_{\odot}$ to 1 $M_{\odot} \cdot$

b) There is a clear relationship between the ionized mass and the electron density: the largest masses occur for nebulae with the lowest density.

c) A given density is correlated with only a small range of mass. Since the range of masses is considerably greater than the errors involved, the range is certainly real.

Comparable data is available for the 'galactic center nebulae'. Because the distance to these nebulae is well known this data will be presented before further discussion.

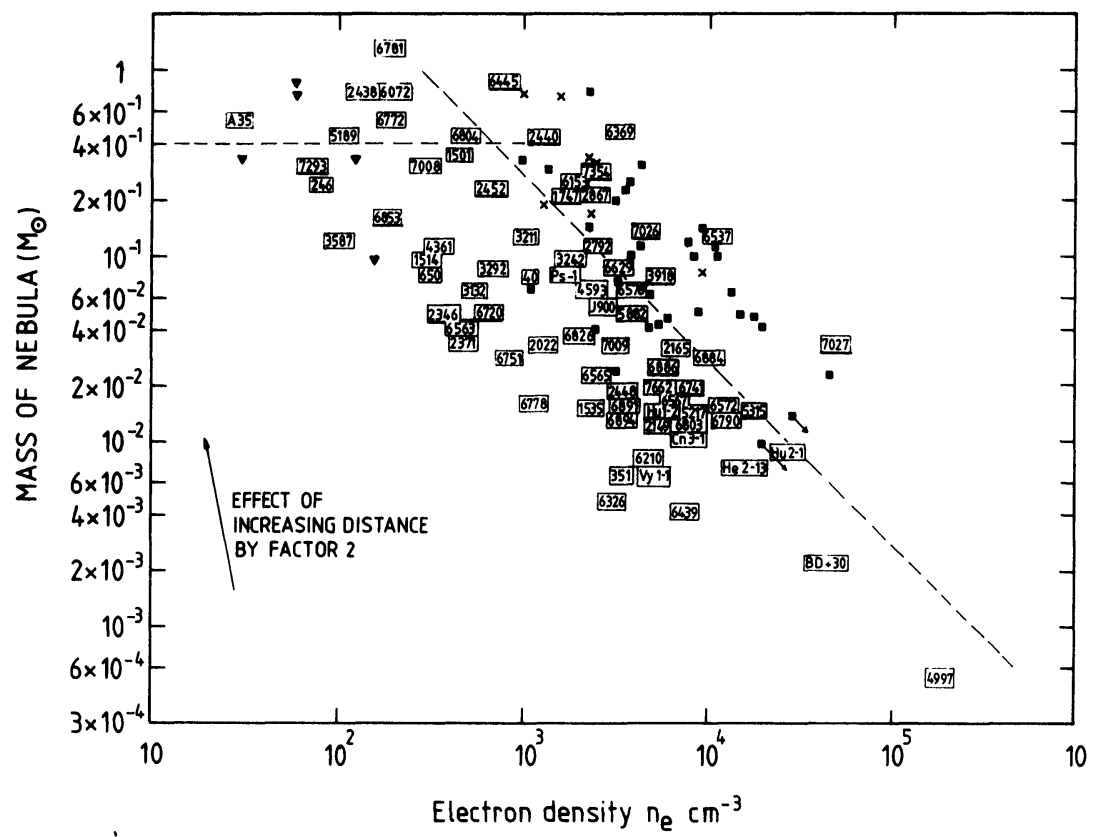

Fig. 4 The rms density is plotted against the mass of the nebula. The nearby nebulae are identified by NGC or other numbers. The filled squares are nebulae near the galactic center and observed with the VLA. The crosses are also galactic center nebula, but observed optically. The triangles are the few resolved nebulae in the Magellanic cloud. The lines are from Cudworth's proper motion study to calibrate distance scales for optically thin (horizontal line) and optically thick nebulae (sloping line).

\section{GALACTIC CENTER NEBULAE}

These nebulae are selected on the basis of two criteria. Firstly, they are within a few degrees of the galactic center on the plane of the sky. Using this criterion alone there is more than $90 \%$ certainty that the nebulae are actually near the center. Secondly, only 
nebulae with very high velocity $\left(\mathrm{V}_{\mathrm{LSR}}>150 \mathrm{~km} \mathrm{~s}^{-1}\right)$ are considered. It is very unlikely that nebulae selected by these criteria will be far from the center.

The distance to the galactic center used is $9 \mathrm{kpc}$. The error is probably less than $1 \mathrm{kpc}$. Because of the distance, many of the nebulae are quite small. The number of optically observed nebulae useful in mass determination is limited, because it is difficult to measure angular sizes of less than 2" accurately with optical telescopes. Radio continuum observations with the VLA have extended the number of nebulae which can be included, since this telescope can measure sizes an order of magnitude smaller. About 40 galactic center nebulae have now been measured with the VLA (Gathier et al., 1983).

The resultant masses and densities for the galactic center nebulae are shown in Fig. 4, as crosses (optical sizes) and filled squares (radio measurements). The masses have approximately the same variation with $n_{e}$ as the nearby nebulae. A difference is that the galactic center nebuale occupy only the upper part of the range of $\mathrm{Ne}$ and $M$ covered by the nearby objects. There are two possible reasons for this:

(a) The lower part of the range for the nearby nebulae is populated because the distance of these objects has been underestimated.

(b) There is a selection effect (discovery of the brightest nebulae) for the galactic center objects which causes one to pick out the more massive nebulae for observation.

While it is difficult to rule out possibility (a), discussion of selection effects in the next section makes it clear that (b) is the most likely explanation.

\section{E. DISCUSSION OF THE NEBULAR MASS VARIATION}

The fact that nebulae have a large range of ionized masses has two possible explanations:

a) The total mass ejected during formation of the nebula also varies over this range, and the ionized mass represents most of the total mass.

b) The total mass is considerably higher than the ionized mass and the ionization is limited because the nebulae are often optically thick in Lyman continuum radiation.

The first 'explanation' is likely to be wrong because it does not explain the variation of mass with density. On the other hand, the second 'explanation', predicts in a simple way just a mass-density relation. Consider a star of constant radiation surrounded by an expanding gas mass. If the gas is optically thick to the ionizing radiation, it absorbs all of the (constant) number of ionizing photons, $K$. In equilibrium, the number of ionizing photons is equal to the number of recombinations in the gas, $\mathrm{K}=\mathrm{n}_{\mathrm{e}}{ }^{2} \Delta \mathrm{v}$, where $\Delta v$ is the ionized volume. The mass ionized is

$$
M_{i} \sim n_{e} \Delta v=K / n_{e}
$$


The mass determined from observation has approximately this same variation with density for the higher density nebulae. We adopt this simple picture as a working hypothesis.

This picture has the important consequence that all nebulae with higher density are optically thick (ionization bounded) in the Lyman continuum. The dividing line is about $\mathrm{n}_{e} \simeq 3 \times 10^{2} \mathrm{~cm}^{-3}$ or

somewhat higher and may be somewhat different for individual nebulae.

A further consequence is that the ionized masses found for the low density nebulae represent the total nebular mass, which appears to vary between $0.1 \mathrm{M}_{\odot}$ and $1 \mathrm{M}_{\odot}$ 。

\section{F. RELATIONSHIP WITH STATISTICAL METHODS FOR DETERMINING DISTANCES}

1. 'Shklovskii method'. This method assumes the ionized nebular mass is constant. It is usually calibrated using the mass of NGC 246 , whose distance is determined from spectroscopic parallax. As can be seen from Fig. 4, the resulting mass for about $60 \%$ of the nebulae is within a factor 5 of this value. In other words, the distance of these $60 \%$ is determined only to within a factor 2. Larger errors occur for the high density nebulae. In some cases this method will lead to distance errors of an order of magnitude.

2. Proper motion studies. Cudworth (1974) has analysed measurements of the proper motions of the central stars of 51 nebulae. The measurements are not individually significant, so that they must be treated statistically. This was done by dividing the nebulae into two groups, those which are optically thin (density bounded) and optically thick (radiation bounded). The division was made on the basis of the nebular size, and is therefore related to the density.

For the optically thin nebulae Cudworth assumed that the nebular mass is constant (as above) and used the proper motion statistics to determine the value of the mass. The result, $M=0.4 M_{\odot}$, is shown as a horizontal line in Fig. 4. It is a better fit to the masses of the low density nebulae. This should be so since the low mass, high density nebulae have been eliminated from this sample.

For the optically thick nebulae Cudworth assumed that the absolute $\mathrm{H} \beta$ flux is constant and determined the value of the constant from the proper motion statistics. The result is:

$$
d=5\left[F_{H \beta}\right]^{1 / 2}
$$

where $d$ is in $\mathrm{Kpc}$ and $\mathrm{F}_{\mathrm{H} \beta}$ is the measured $\mathrm{H} \beta$ flux in units of $10^{-11} \mathrm{erg}$ $\mathrm{cm}^{-2} \mathrm{~s}^{-1}$. This result is also plotted as a sloping line in Fig. 4. The agreement is considerably better than the constant mass assumption for nebulae having $\mathrm{n}_{e}>6 \times 10^{2} \mathrm{~cm}^{-3}$. The precise position of the line in the diagram (or the constant in the above equation) is quite uncertain, since only 17 nebulae are involved in Cudworths study. Furthermore, the assumption of a unique absolute $\mathrm{H} \beta$ flux for all optically thick nebulae is a poor approximation (see below and Fig. 5). 
G. ON THE DISPERSION OF THE POINTS IN FIG. 4 .

The dispersion of the points in the mass-density diagram is larger than would be expected from the errors involved. Since the distance of individual objects is often uncertain, sometimes by as much as a factor 2, this must be a contributing factor. However there are reasons to think that it is not the overriding factor. The first is that for a given density in the optically thick region, the masses appear to vary by about a factor of 50. Such a large variation is well in excess of what the uncertainties in the distance would contribute. Secondly, the nebulae at the galactic center show a large dispersion, although it is only a factor of 20. This dispersion is clearly real because the nebulae are essentially all at the same distance.

The reason for the (smaller) dispersion in the optically thin region is clear: the (intrinsic) nebular masses vary by a factor of between 3 and 10 . The reason for the dispersion in the optically thick

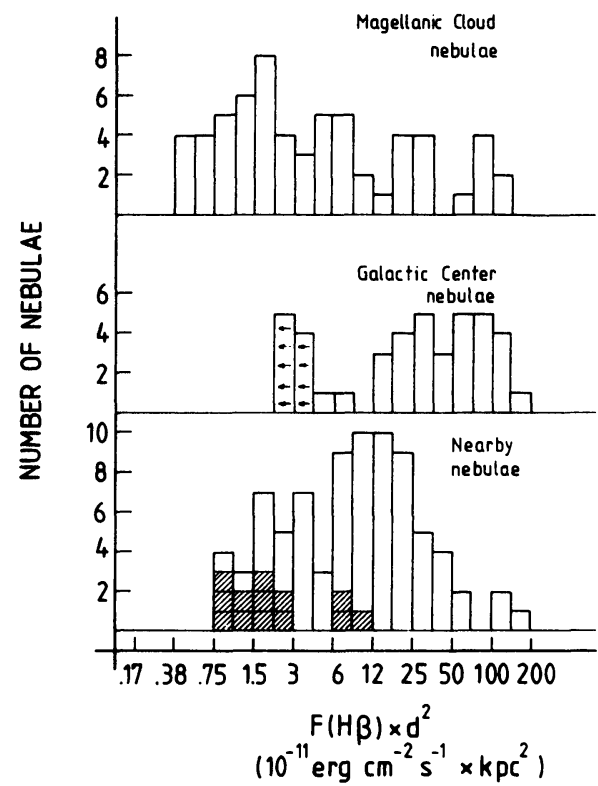

\section{$\underline{\text { Fig. } 5}$}

Histograms of intrinsic $\mathrm{H} \beta$ flux.

(a) Magellanic cloud nebulae (Jacoby, 1980)

(b) Galactic center nebulae used in Fig. 4 (Gathier, et al., 1983).

(c) Nearby nebulae (shaded areas are optically thin nebulae).

region is different. It can best be understood by recalling that the sloping line in Fig. 4 is the locus of nebulae with a constant intrinsic value of $\mathrm{H} \beta$ flux. Other values of intrinsic $\mathrm{H} \beta$ flux will appear as parallel lines, small H values to the left, larger values to the right. The dispersion in the optically thick nebulae can thus be explained by a large spread in the intrinsic $\mathrm{H} \beta$ flux.

There is other evidence for such a spread in the intrinsic flux. Recently, Jacoby (1980) made a very deep survey of selected regions in the Magellanic clouds and measured the integrated flux of the nebulae found. The results are shown in Fig. 5a, which is a histogram of the intrinsic $\mathrm{H} \beta$ flux (measured flux multiplied by $\mathrm{d}^{2}$ ). It can be seen that the intrinsic flux varies by a factor of 400 ! For comparison, 
Fig. 5c shows the intrinsic flux of the nearby nebulae discussed earlier. These can be divided into optically thin (shown as striped area) and optically thick nebulae. As can be seen, the optically thin nebulae all have small intrinsic $\mathrm{H} \beta$ flux. The low end of the distribution of nearby nebulae does not extend to such small values as is the case in the Magellanic clouds, and is probably not the result of a systematic overestimate of the distances of some of the nearby nebulae. This discrepancy is due to the fact that most of the nearby faint nebulae were not included in our sample, because they lie at high galactic latitudes and an independent value of the distance cannot be obtained for them. Fig. 5 includes only a few nebulae with diameters greater than 100 ", whereas more than 50 are known to exist!

Fig. 5b shows the distribution of intrinsic $\mathrm{H} \beta$ flux (really converted radio flux) of the galactic center nebulae in our sample. It does not extend to low intrinsic $\mathrm{H} \beta$ values, presumably because only the brighter objects have been selected for observation. This is evidence for the earlier statement that the lack of galactic center nebulae in the lower left side of Fig. 4 was a selection effect.

The mass-density dispersion of the optically thick nebulae is correlated with a very large variation in the intrinsic $H \beta$ flux. The variation in flux is a direct consequence of intrinsic differences in the number of ionizing photons, which in turn follows from differences in radius and temperature of the central star.

\section{H. THE EFFECTIVE TEMPERATURES OF THE CENTRAL STAR}

There are a number of methods of determining the effective temperatures. The most important are:

1) Use of the spectral type for those stars with a normal 0 or of type spectrum, which are generally the relatively cooler stars.

2) Zanstra temperatures

3) Comparison of the continuum emission between the visual and the ultraviolet ( $\lambda 1300 \AA$ ) with either a blackbody or a model atmosphere.

4) Energy balance or Stoy method.

Method 1) may only be applied to a limited number of central stars, whose spectra mimic quite closely the well studied normal 0 type stars. At present, model atmospheres are being constructed to reproduce the oberved spectra of higher temperature stars which have it absorption line spectra (Mendez et al., 1981), which 11 increase the usefulness of this method.

The application of method 2) has been questioned for some time for the following reason. The method can be applied using either the hydrogen lines or the lines of ionized helium. The ratio of the flux shortward of $\lambda 912 \AA$ to the visual continuum is then computed. The ratio is then compared with that expected from a blackbody. The temperature is assumed to be that of the blackbody with the same ratio. The difficulty occurs because in a substantial number of stars the values of $\mathrm{T}_{z}(\mathrm{H})$ and $\mathrm{T}_{z}$ (HeII) are not the same; the latter value is consistently higher than the former.

There are two possible explanations for this difference. 
Firstly the nebula may be optically thin in the radiation field which can ionize hydrogen. Since some photons which ionize hydrogen would then not contribute to the ratio, $T_{z}(H)$ would only be a lower limit, and $\mathrm{T}_{z}$ (HeII) would be a better approximation. Secondly, it may not be correct to use a blackbody for comparison, but instead an atmosphere with emission in excess of that of a blackbody shortward of $\lambda=228 \AA$. Several of the models of Mendez et al.(1981) and Wesemael et al.(1980) have this characteristic. This would result in $\mathrm{T}_{z}(\mathrm{H})$ being the more nearly correct value and $T_{z}$ (HeII) being excessively high.

Which of these two reasons is correct? In the last two sections it has been argued that Fig. 4 may be used to separate the optically thick nebulae from those that are optically thin. For the optically thick nebulae it is reasonable to assume that the second reason is correct and that $T_{z}(H)$ is a good approximation to the effective temperature. The question still arises as to whether $T_{z}(H)$ should be computed by comparing with a blackbody or with a model atmosphere. The remarkable fact is that for the three series of model atmospheres in the literature (Hummer and Mihalas, 1970; Wesemael et al., 1980; Mendez et al. 1981), the same temperature is obtained (within 10\%), regardless of whether a blackbody or a model atmosphere is assumed. This is only true for $T_{z}(H)$; it is definitely not true for $T_{z}$ (HeII). The use of the atmosheric models of Hummer and Mihalas (1970) give a higher value of $\mathrm{T}_{z}$ (HeII) than a blackbody atmosphere (making the discrepancy worse). However the pure hydrogen models of Wesemael et al.(1980) and several of the models of Mendez et al.(1981) do just the opposite, lowering $\mathrm{T}_{z}$ (HeII), even to values lower than $\mathrm{T}_{Z}(\mathrm{H})$. More accurate models are only a hope for the future, but it seems likely that $\mathrm{T}_{Z}(\mathrm{H})$ will be relatively unaffected.

For the optically thin nebulae, method 3), comparison of continuum emission, must be applied. It can only be used when both visual and ultraviolet measurements are available because of the small change in slope with temperature of a blackbody curve for both objects. Several problems arise in this method. First of all, the extinction must be well determined because correction greatly affects the slope of the continuum. Results for heavily reddened nebulae are therefore less reliable. Secondly, current ultraviolet observation include the nebular continuum with the stellar continuum and the separation is often difficult. A counter-balance to these difficulties is that the interpretation is more straightforward. A blackbody and the model atmospheres mentioned above all predict approximately the same slope between $\lambda=$ $1300 \AA$ and $\lambda=6000 \AA$.

One of the difficulties mentioned above applies also to method 2). For the smaller nebulae it is sometimes very difficult to separate the stellar continuum from the nebular continuum. The problem is greatest for the hotter stars, because relatively more flux is used for nebular ionization and consequently produces more nebular continuum. Careful observational work is beginning (e.g. Kohoutek and Martin, 1981; Martin, 1981) but when $90 \%$ of the observed continuum emission is nebular, the resulting magnitude $\mathrm{m}_{\mathrm{v}}$ or stellar continuum must be considered unreliable. This is particularly true of the very hot central stars of the nebulae Peimbert calls Type I, which have extreme- 
1y high helium and nitrogen abundances and a strong concentration to the galactic plane, e.g. NGC 2440, 6302, 6445, 6741 and 7027 .

For these hot, faint stars the most reliable temperatures can be obtained from method 4 , the energy balance or Stoy method, which measures the excess energy of each lonizing photon. It is based on the measurement of the ratio of energy emitted in collisionally excited lines to that emitted in $\mathrm{H} \beta$. The helium abundance and state of ionization must also be known, but the magnitude of the star does not have to be known. A recent discussion of this method, together with resultant temperatures, has been given by Preite-Martinez and Pottasch (1983). Some resultant stellar temperatures are given in Table 4. When the temperatures can be compared to those determined from other methods, the agreement is good.

TABLE 4 - STELLAR TEMPERATURES FROM ENERGY BALANCE METHOD

\begin{tabular}{|c|c|c|c|c|c|}
\hline NEBULA & $\frac{\sum I(\operatorname{col} 11 \cdot \text { exc. })}{I(H \beta)}$ & $\mathrm{T}$ & NEBULA & $\frac{\sum I(\operatorname{col} 11 \cdot e x c \cdot)}{I(H \beta)}$ & $\mathrm{T}$ \\
\hline $\begin{array}{rr}\text { NGC } & 40 \\
\text { IC } & 418 \\
\text { NGC } & 6826 \\
6572 \\
\\
1535 \\
3918 \\
\\
7662\end{array}$ & $\begin{array}{r}9.5 \\
8.5 \\
11.3 \\
19.0 \\
22.4 \\
38.4 \\
36.1\end{array}$ & $\begin{array}{r}36.000 \mathrm{~K} \\
33.000 \\
41.000 \\
60.000 \\
66.000 \\
105.000 \\
100.000\end{array}$ & $\begin{array}{r}2165 \\
3211 \\
6886 \\
6741 \\
2440 \\
7027 \\
6302\end{array}$ & $\begin{array}{l}41.7 \\
47.9 \\
55.4 \\
66.9 \\
70.8 \\
73.6 \\
92.7\end{array}$ & $\begin{array}{l}115.000 \mathrm{~K} \\
135.000 \\
165.000 \\
230.000 \\
250.000 \\
270.000 \\
350.000\end{array}$ \\
\hline
\end{tabular}

I. POSITION OF THE STARS ON THE HR DIAGRAM.

When the distances and effective temperatures have been calculated only the luminosity must be found in order to place the stars on the HR diagram. The usual method for determining luminosity is to combine the temperature, visual magnitude $\mathrm{m}_{\mathrm{v}}$, and distance to obtain the stellar radius. The radius is only slightly dependent on the temperature for these hot objects. The radius is also little affected by the model atmosphere (or blackbody) used, to within about $20 \%$. The radius and temperature then define the luminosity.

The greatest source of error for the hotter objects is the measurement of $\mathrm{m}_{\mathrm{v}}$ as has been pointed out in the discussion of ste1lar continuum. This is probably why the hot objects listed above are not often plotted on the HR diagram. For optically thick nebulae the luminosity can be found without knowing the visual magnitude. This is because all the stellar radiation shortward of $\lambda=912 \AA$ is absorbed in the nebula and transformed into (nebular) radiation longward of $\lambda=$ $912 \AA$. All of this radiation can be measured, when ultraviolet and infrared observations above the atmosphere are available. The luminosity is thus a well determined quantity (its greatest uncertainty is the distance determination).

Even when far ultraviolet and infrared observations are not available, the luminosity can be reasonably well approximated as 100 
times the luminosity in the $\mathrm{H} \beta$ 1ine. This works empirically for such different objects as NGC 7027 and the low stellar temperature nebulae $\mathrm{BD}+30$ 3639. In NGC 7027 the ratio of forbidden emission lines to $H \beta$ is much higher than in $B D+303639$, but this is compensated for in the latter object by the much higher stellar flux which is directly emitted longward of $\lambda=912 \AA$.

For optically thin nebulae this method will not work and one is dependent on calculating the radius using $\mathrm{m}_{\mathrm{v}}$. Since most of these nebulae are larger the influence of nebular emission in a small diaphragm is minimal.

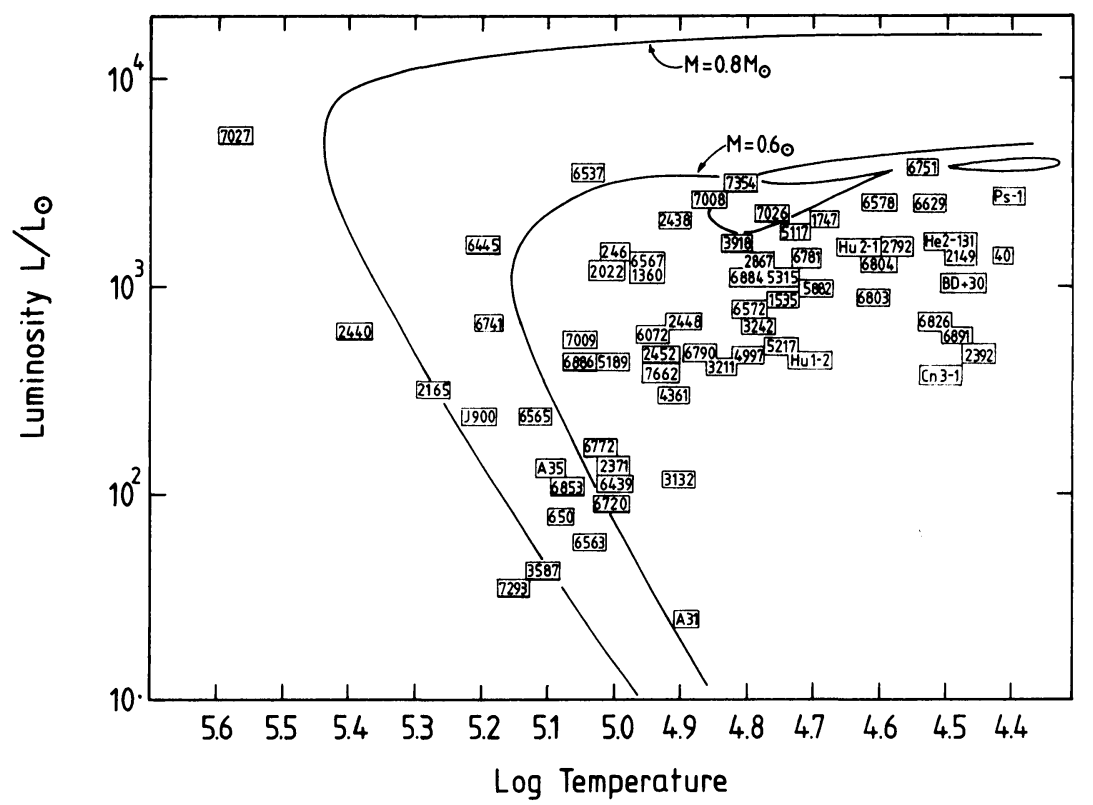

Fig. 6 Luminosity of the central star is plotted against temperature. Nebulae are identified by their NGC, etc., number. Those with high helium or nitrogen abundance are underlined.

The resultant $\mathrm{HR}$ diagram is shown as Fig. 6. In the diagram each star is identified by the NGC, etc., number of the nebula in which it is found. The stars underlined are those where the nebulae are helium and nitrogen or carbon rich. For orientation and comparison, the theoretical evolutionary tracks calculated by Paczynski (1971) are given for $0.6 \mathrm{M}_{\odot}$ and $0.8 \mathrm{M} \odot$. The lower temperature stars appear to all have luminosities between $3 \times 10^{2} \mathrm{~L}_{\odot}$ and $5 \times 10^{3} \mathrm{~L}_{\odot}$. This range seems to remain fixed independent of stellar temperature. For stellar temperatures greater than $80.000 \mathrm{~K}$ much lower luminosities are observed as well, extending to almost $20 \mathrm{~L}_{\odot}$.

As evolution progresses, the temperature and luminosity of the star change. Thus the time may be considered as an additional parameter, which may even be a measureable quantity: the nebular radius 
divided by the expansion velocity. Schonberner (1981) has plotted the absolute visual magnitude of the central star against this time $t$ and compard this to theoretical expectation. He points out that the major uncertainty is distance. He has used the distances determined by assuming a constant nebular mass of $M=0.2 M_{\odot}$. Fig. $7 a$ is a plot of $M_{V}$ against $t$ using this assumption. It differs from the plot made by Schonberner, presumably because of a somewhat different selection of nebulae (those plotted in Fig. 6) and an observed value of the expansion velocity is used here instead of an assumed constant value of 20 $\mathrm{km} \mathrm{s}^{-1}$. Schonberner's plot shows less spread. He compares it to the theoretical curves, shown as solid lines in Fig. 7, and concludes that the central stars have a rather narrow range of mass.
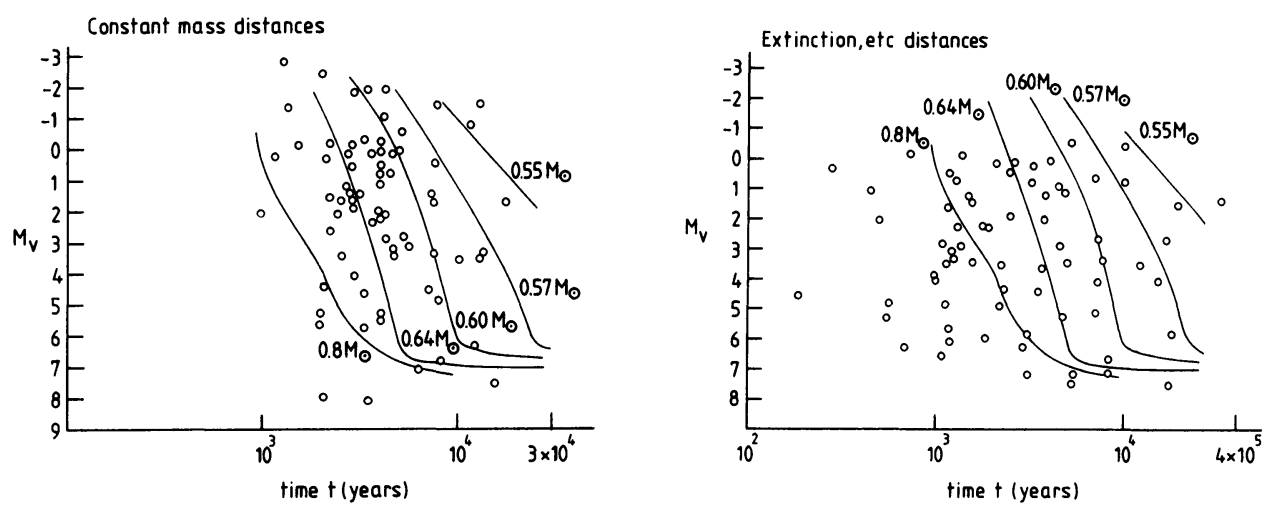

Fig. 7 Absolute visual magnitude is plotted against the nebular age $t$ (in years), defined as the radius of the ionized region divided by the expansion velocity. Theoretical expectations for different masses are plotted as solid lines (Schonberner, 1981, except $M=$ $0.8 M_{\odot}$ taken from Paczynski, 1971).

(a) Distance determined from assumption of constant nebular mass.

(b) Distance determined by independent methods.

Fig. 7b is the same plot ( $M_{v}$ against $t$ ) but now using the distances we have described above. The greatest difference is the much larger spread in $t$, extending down to a few hundred years. One might conclude that this is inconsistent with Fig. 6 where it appears that most central stars have masses less than $0.6 \mathrm{M}_{\odot}$. The difficulty is that the age or time cannot be computed from the ratio of the ionized size to velocity for an optically thick nebula, because the ionized size is smaller than the actual size. All the nebulae known to be optically thin are at the right side of Fig. $7 \mathrm{~b}$ with $t>3 \times 10^{3}$ years.

Schonberner's conclusion appears correct, however. It is easier to see from a comparison to the observations and theoretical expectation in Fig. 6, where one would conclude that most of the stellar masses are in the range $0.5 \mathrm{M}_{\odot}$ to $0.6 \mathrm{M}_{\odot}$. Some of the very hot stars may have masses exceeding this, possibly $0.8 \mathrm{M}_{\odot}$ or somewhat higher. 


\section{CONCLUDING REMARKS}

An important change has occurred in the last 15 years in the position of the central stars in the HR diagram. Earlier the luminosity was thought to extend to $10^{4}$ to $10^{5} \mathrm{~L}_{\odot}$, and to increase from the low temperature end to a maximum value near $70.000 \mathrm{~K}$ (the Harman Seaton track). Now it appears that the luminosity is lower, within a factor 3 of $10^{3} \mathrm{~L}_{\odot}$, and it remains constant for a large part of the temperature range, until it begins to decrease at $10^{5} \mathrm{~K}$. What has caused this large change? While many factors are involved, it is primarily the growing realization that the large majority of nebulae are optically thick in Lyman continuum radiation. This has three consequences. First of all, the rather large correction for radiation not absorbed by the nebulae is no longer needed. Secondly, the temperature of some of the nebulae is lowered. Thirdly, the distance of some of the smaller nebulae is found to be considerably nearer.

The lower luminosity for the beginning phases of the central star, coupled with the realization that the mass probably lies within the range $0.5 \mathrm{M}_{\odot}$ to $0.7 \mathrm{M}_{\odot}$ provide a basis for further work on the prenebular phase of evolution.

\section{REFERENCES}

Acker, A., 1976, Publ. Obs. Astron. Strasbourg, Vol. 4, fasc. 1. Acker, A., 1978, Astron. Astrophys. Supp. 33, 367. Chudovicheva, 0., 1964, Iz. Pulkova, Obs. 23, 154. Cudworth, K.M., 1974, Astron., J., 79, 1384.

Gathier, R., Pottasch, S.R., 1983.

Gathier, R., Pottasch, S.R., Goss, W.M., v. Gorkom, J.H., 1983.

Harris, W.E., 1976, Astron. J. 81, 1095.

Hummer, O.G., Mihalas, D., 1970, Mon. Not. Roy. Astron. Soc., 147, 339. Jacoby, G.H., 1980, Astrophys. J. Supp. 42,1.

Jacoby, G.H., 1981, Astrophys. J. 244, 903.

Kohoutek, L., Martin, W., 1981, Astron. Astrophys. 94, 365.

Latypov, A.A., 1955, Pubi. Astron. Obs. Tashkent, 5,31.

Liller, M.H., Liller, W., 1968, Plan. Neb., IAU Symp. 34, ed. Osterbrock, D., O'Dell, C.R. (Reidel, Dordrecht).

Liller, M.H., Welther, B.L., Liller, W., 1966, Astrophys. J., 144, 280. Lucke, P.B., 1978, Astron. Ástrophys., 64, 367.

Lutz, J.H., 1977, Astron. Astrophys., 60, 93.

Lutz, J.H., 1978, Plan. Neb. IAU Symp. 76, Ed. Y. Terzian (Reidel, Dordrecht).

Martin, W., 1981, Astron. Astrophys., 98, 328.

Mendez, R., 1978, Mon. Not. Roy, Astron. Soc., 185, 647.

Mendez, R.H., Kudritzki, R.P., Gruschinski, J. Simon, K.P., 1981, Astron. Astrophys., 101, 323.

Pazynski, B., 1971, Acta Astron., 21, 417.

Pottasch, S.R., Gathier, R., Goss, W.M., 1983.

Pottasch, S.R., Goss, W.M., Arnal, E.M., Gathier, R., 1982, Astron. Astrophys., 106, 229.

Preite-Martinez, A., Pottasch, S.R., 1983.

Schoenberner, D., 1981, Astron. Astrophys., 103, 119.

Wesemael, F., Auer, L.H., Van Horn, H.M., Savedoff, M.P. 1980, Astrophys. J. Supp., 43, 159. 
RODRIGUEZ: When using the extinction method, do you apply any correction for possible internal extinction?

POTTASCH: Present evidence suggests that, for most nebulae, a correction for internal extinction is not necessary. For many nebulae, the extinction may be determined by several methods: (a) comparison of radio and $H B$ emission; (b) $\lambda 2200$ feature; (c) Balmer decrement; (d) He II $\lambda 4686 / \lambda 1640$ ratio. A11 these methods give the same extinction, when interpreted with a "standard" interstellar extinction curve. If a substantial part of the extinction arose from internal nebular dust, one would not expect this good agreement because the internal dust is expected to have properties which are substantially different from the interstellar dust.

OSTERBROCK: The progress in getting distances of PN shown in this work is very gratifying. It is, therefore, important to make quantitative estimates of the accuracy of these distances. The ideal case for the extinction method would be when all the stars were exactly along the line of sight to the $\mathrm{PN}$ and their intrinsic colors were exactly known. In practice, there is some scatter in angle and the intrinsic colors are not precisely known. You could evaluate the errors in the extinction method by applying it to a few stars of accurately known distance, in fields of about the same richness as the PN fields. Have you done anything like this, or do you intend to do so? Can you give any numerical estimate of the probable errors in the extinction method? POTTASCH: We are in the process of analysing the errors involved in this method. The errors depend on many factors and will vary from nebula to nebula. If extensive observations have been made in the region close to the nebula (within $1^{\circ}$ ) and stars are observed with extinctions $50 \%$ higher than the nebular extinction, the extinction distance is probably accurate to within $30 \%$. At the other extreme, when it is necessary to use observations as far away as $5^{\circ}$ or even $10^{\circ}$ from the nebula, the distance derived may be within only a factor 2 of the correct value.

ALLER: The level of excitation of NGC 6741 resembles that of NGC 7662 and our theoretical models give $T_{*} \approx 95000 \mathrm{~K}$. We could not reproduce the observed spectrum with a stellar flux corresponding to $\mathrm{T}_{*} \approx$ $200000 \mathrm{~K}$, as you suggest.

POTTASCH: The ratio of the energy in collisionally excited lines to $\mathrm{H} \beta$ is twice as high in NGC 6741 as in NGC 7662. This observational fact can most easily be explained if the exciting star of NGC 6741 has a considerably higher temperature than that of NGC 7662 .

WEIDEMANN: In your HR diagram, the luminosities of the lower temperature objects cluster around $10^{3} \mathrm{~L}_{0}$. Although this behaviour is qualitatively what is expected from evolutionary calculations (low mass nuclei spend most of their life at high luminosity, high mass nuclei at lower luminosity), your result is quantitatively difficult to understand nuclei of $0.55 \mathrm{M}_{\odot}$ already reside at $10^{3.2} \mathrm{~L}_{\Theta^{-}}$I conclude that either the distances are still underestimated or the temperatures are too low. The smaller distances would present the additional problem of a higher PN birth rate and increase the existing discrepancy with the White Dwarf birth rate (cf. Weidemann, 1977, Astron. Astrophys. 61, L27). 
POTTASCH: The distances of only the optically thick nebulae in my sample are smaller than previously adopted values, and then by only about $50 \%$, on the average, as compared with Cudworth's values. The value of the local birthrate of $\mathrm{PN}$, on the other hand, is mainly determined by the distances of the large, optically thin nebulae, and I presented evidence that their distances have probably been underestimated in previous studies.

COHEN: I have the impression that, when the observed infrared contribution is included, a number of nuclei are as luminous at $10^{4} \mathrm{~L}_{\odot}$ : Are we selecting the highest mass objects or using incorrect distances? POTTASCH: I have checked that the luminosities plotted in the HR diagram exceed the total observed luminosity, including the infrared

contribution. The high luminosity which you quote, especially for $\mathrm{BD}+30^{\circ} 3639$, arises from using too large a value for the distance. 